\title{
Equidade e acesso aos cuidados em saúde mental em três estados nordestinos
}

\author{
Equity and access to mental health care in three Northeastern states
}

\author{
Magda Dimenstein (https://orcid.org/0000-0002-5000-2915) ${ }^{1}$ \\ Ana Carolina Rios Simoni (https://orcid.org/0000-0001-9805-6966) ${ }^{1}$ \\ João Paulo Macedo (https://orcid.org/0000-0003-4393-8501) ${ }^{2}$ \\ Natália Nogueira (https://orcid.org/0000-0002-3827-8257) ${ }^{1}$ \\ Bianca Caroline Noronha Sousa Barbosa (https://orcid.org/0000-0002-6586-8121) ${ }^{1}$ \\ Brisana Índio do Brasil de Macêdo Silva (https://orcid.org/0000-0003-1150-8291) ${ }^{3}$ \\ João Batista do Amaral Filho (https://orcid.org/0000-0002-0306-614X) ${ }^{1}$ \\ Roberto Cirilo de Araújo Silva (https://orcid.org/0000-0002-9555-7658) ${ }^{1}$ \\ Mariana Tavares Cavalcanti Liberato (https://orcid.org/0000-0002-9410-0437) ${ }^{3}$ \\ Caio Lucas do Carmo Prado (https://orcid.org/0000-0002-5853-5599) ${ }^{3}$ \\ Mateus Villarroel Alcantara Saraiva Leão (https://orcid.org/0000-0002-1585-1890) ${ }^{3}$ \\ Bruna Almeida Quinto (https://orcid.org/0000-0003-2272-8580) ${ }^{3}$ \\ Leilany Ferreira Soares (https://orcid.org/0000-0001-7742-7414) ${ }^{3}$
}

${ }^{1}$ Universidade Federal do Rio Grande do Norte. Av Senador Salgado Filho s/n, Lagoa Nova. 59078970 Natal RN Brasil. mgdimenstein@gmail.com ${ }^{2}$ Universidade Federal do

Delta do Parnaíba. Parnaíba PI Brasil.

${ }^{3}$ Universidade Federal do

Ceará. Fortaleza CE Brasil.

\begin{abstract}
The scope of this study is to discuss equity and access to mental health care by means of analysis of the distribution of primary care teams (Community Health Agents; Family Health Teams; the Expanded Family Health Nucleus) and the coverage of the Psychosocial Care Network in three Northeastern states (Ceará, Piaui and Rio Grande do Norte) and their respective health regions. It is a descriptive study, supported by exploratory quantitative analysis. For this purpose, the database of the Department of $\mathrm{Ba}$ sic Care/DATASUS was used to collect secondary data in relation to the historical trajectory of training of Primary Care teams. Regarding the points of care of RAPS, the database made available by the National Mental Health Coordination of the Ministry of Health was consulted. The conclusion of the investigation was that prior to 2018 there was a major drive in the country into the interior and expansion of coverage in relation to primary and psychosocial care, impacting the expansion of equity in territories of lesser economic and social development. However, it was also observed that Psychosocial Care Network services are more prevalent in the interior while the other related services are more developed in capitals and large municipalities.
\end{abstract}

Key words Equity in coverage, Accessibility of health services, Primary health care, Psychosocial care, Mental health
Resumo Objetiva-se discutir equidade e acesso em saúde mental por meio da análise da distribuição das equipes da atenção primária (Agentes comunitários de Saúde; Equipes de saúde da Família; Núcleo Ampliado de Saúde da Família) e da cobertura da Rede de Atenção Psicossocial (RAPS) em três estados nordestinos (Ceará, Piauí e Rio Grande do Norte) nas suas respectivas regiões de saúde. Trata-se de um estudo descritivo, apoiado por análise quantitativa exploratória. Para tanto, recorremos à base de dados do Departamento de Atenção Básica/DATASUS para a coleta dos dados secundários em relação às séries históricas de habilitação das equipes da Atenção Primária. Quanto aos pontos de atenção da RAPS, utilizamos a base de dados disponibilizada pela Coordenação $\mathrm{Na}$ cional de Saúde Mental do Ministério da Saúde. A investigação concluiu que até 2018 observou-se no país um importante movimento de interiorização e expansão da cobertura em relação à atenção primária e psicossocial, impactando na ampliação da equidade nos territórios de menor desenvolvimento econômico e social. Contudo, verificou-se que os serviços de APS estão mais interiorizados, enquanto os demais dispositivos da RAPS estão mais desenvolvidos nas capitais e grandes municípios. Palavras-chave Equidade em cobertura, Acessibilidade aos serviços de saúde, Atenção Primária em Saúde, Atenção psicossocial, Saúde mental 


\section{Introdução}

O Sistema Único de Saúde (SUS), enquanto política de Estado, tem reconhecimento internacional por afirmar a saúde como direito universal e pelo grau de abrangência que estrutura suas políticas, programas, serviços e equipes de saú$\mathrm{de}^{1}$. Os níveis de repostas alcançados no Brasil, ao longo de mais de 30 anos do SUS, em relação ao controle do HIV-AIDS, cobertura vacinal, tabagismo e com a capilaridade das equipes da atenção primária à saúde e atenção psicossocial, dentre outras ações, indicam avanços importantes na diminuição das desigualdades históricas em saúde com políticas públicas mais equânimes, inclusive, com a adoção de políticas de promoção de equidade em saúde, incorporando segmentos antes excluídos como a população indígena, negra, do campo, das águas e das florestas e comunidade LGBTQi².

No entanto, nos últimos anos, a despeito dessas conquistas, observa-se o desmonte de duas importantes políticas estruturantes no âmbito da saúde para a redução do quadro de iniquidades em saúde no território brasileiro. Trata-se da alteração da Política Nacional da Atenção Básica (PNAB) efetuada em 2017, desfigurando a lógica matriz da organização da atenção primária no Brasil, baseada na conjugação entre necessidades de saúde, territorialização, adscrição de clientela, vínculo e responsabilidade sanitária. A característica central de uma atenção primária forte é o acesso aos cuidados, pela entrada de indivíduos, famílias e comunidades no sistema de saúde e disponibilização de serviços e recursos de prevenção, promoção, recuperação e reabilitação da saúde em determinado tempo/espaço ${ }^{3}$. A mudança no parâmetro de financiamento, acompanhado da flexibilização e redução da carga horária dos profissionais das Equipes de Saúde da Família (EqSF) e Saúde Bucal (SB) e o desmonte do Núcleo Ampliado de Saúde da Família (NASF) estão transformando a Atenção Básica em uma atenção seletiva, com pacotes de serviços e programas focalizados e ações baseadas na queixa-conduta.

Do mesmo modo, desde 2016, registramse retrocessos importantes no âmbito da Política Nacional de Saúde Mental, Álcool e outras Drogas, com o objetivo de remanicomialização do cuidado em saúde mental, cujas normativas foram compiladas na Nota Técnica 11/2019-CGMAD/DAPES/SAS/MS, conhecida por "Nova Política Nacional de Saúde Mental"4. Desde então, tem havido incentivo à abertura de leitos e à internação em hospitais psiquiátricos, a retomada dos ambulatórios de saúde mental, acompanhado do fortalecimento do financiamento das Comunidades Terapêuticas em detrimento dos CAPS ad, Unidades de Acolhimento e demais dispositivos da RAPS 5 .

O desmonte orquestrado de ambas as políticas públicas, que visam à redução ou eliminação das diferenças advindas de fatores considerados evitáveis e injustos, favorecendo oportunidades mais equânimes ${ }^{6}$ representa mais um obstáculo ao aprofundamento da equidade em saúde no Brasil. Em um país com diferenças marcantes e permeado de desigualdades estruturais e regionais, as iniquidades em saúde persistem, exigindo que sejam enfrentadas intersetorialmente em sua complexidade. Nesse sentido, faz-se necessário investimentos para qualificar tanto a oferta de serviços (capacidade instalada) e os recursos humanos, quanto ampliar o acesso para melhorar os indicadores da situação de saúde e as condições de vida da população ${ }^{1}$.

Isto posto, apontamos o quão desafiante é fazer avançar o princípio da equidade em saúde, a fim de fazer valer o acesso universal e mais igualitário no SUS, focando não apenas na ampliação da cobertura dos serviços, mas na coordenação das linhas de cuidado e redes de atenção, de forma regionalizada, na realidade de um país com dimensões continentais como o Brasil, cujo o perfil econômico e de porte populacional da maioria dos municípios demanda para a atenção em saúde uma qualificada articulação regional ${ }^{7}$. Assim, corroboramos com Duarte et al. ${ }^{8}$, que a organização do Sistema Único de Saúde em redes regionalizadas é uma condição imprescindível para a garantia de uma oferta eficiente e um passo necessário para alcançar os princípios da equidade e integralidade em saúde.

Contudo, quando se trata da Rede de Atenção Psicossocial, no que diz respeito à acessibilidade e cobertura dos serviços, o cenário é ainda mais nebuloso. Há poucos estudos sobre as necessidades em saúde mental, oferta e organização da atenção a partir do processo de institucionalização da RAPS e regionalização em saúde, em especial no nordeste brasileiro, região do país que concentra inúmeras desigualdades sociais e iniquidades em saúde.

Desta feita, objetivamos com o presente estudo discutir equidade e acesso em saúde mental por meio da análise da distribuição das equipes da atenção primária (Agentes comunitários de Saúde; Equipes de saúde da Família; Núcleo Ampliado de Saúde da Família) e da cobertura 
Trata-se de um estudo descritivo, apoiado por uma análise quantitativa exploratória. Compreendendo que a RAPS é estruturada em sete componentes da atenção, a análise centrou-se em traçar o panorama da cobertura assistencial referente às equipes de ACS, ESF e NASF (Atenção Primária), à rede de serviços tipo CAPS (Atenção Psicossocial Especializada), aos serviços do tipo Comunidades Terapêuticas (Atenção Residencial de Caráter Transitório) e serviços de leitos de internação (Atenção Hospitalar), nos estados do Ceará (CE), Piauí (PI) e Rio Grande do Norte (RN). De acordo com a Portaria n. ${ }^{\circ} 3.088 / 2011$, que deu origem à RAPS, atualmente substituída pela Portaria no 3.588/2017, a rede de atenção psicossocial é composta pelos seguintes componentes: atenção primária, atenção psicossocial especializada, atenção de urgência e emergência, atenção residencial de caráter transitório, atenção hospitalar, estratégia de desinstitucionalização e reabilitação psicossocial. Para produção das informações recorremos à base de dados do Departamento de Atenção Básica/DATASUS para a coleta dos dados secundários em relação às séries históricas de habilitação das equipes da Atenção Primária. Quanto aos demais pontos de atenção da RAPS, recorremos à base de dados disponibilizada pela Coordenação Nacional de Saúde Mental do Ministério da Saúde. Todas as informações produzidas tiveram como referência dezembro de 2018.

Para organizar tais informações considerando o processo de divisão das regiões de saúde em cada estado, apoiamo-nos no modelo de agrupamento (cluster analysis), proposto por Viana et al. ${ }^{9}$. Para isso, os autores analisaram as 438 regiões de saúde que comportam os 5.570 municípios brasileiros, levando em conta o desenvolvimento socioeconômico, a oferta e a complexidade dos serviços de saúde no contexto regional. O modelo propôs então pelo menos cinco grandes conjuntos de regiões de saúde, assim agrupadas: Grupo 1 (baixo desenvolvimento socioeconômico e baixa oferta de serviços) conta com 175 regiões, 2.151 municípios e $22,5 \%$ da população brasileira, localizadas predominantemente no Nordeste; Grupo 2 (médio/alto desenvolvimento

socioeconômico e baixa oferta de serviços) conta com 47 regiões, 482 municípios e 5,7\% da população, localizadas em sua maioria nas regiões Centro-Oeste, Sudeste e Norte; Grupo 3 (médio desenvolvimento socioeconômico e média/alta oferta de serviços) conta com 129 regiões, 1.891 municípios e 20,3\% da população, localizadas predominantemente nas regiões Sudeste e Sul; Grupo 4 (alto desenvolvimento socioeconômico e média oferta de serviços) conta com 27 regiões, 300 municípios e 10,6\% da população, localizadas em sua grande maioria na região Sudeste; Grupo 5 (alto desenvolvimento socioeconômico e alta oferta de serviços) conta com 60 regiões, 746 municípios e 40,9\% da população, localizadas predominantemente nas regiões Sudeste e Sul.

A análise aqui proposta centrou-se nos problemas referentes à cobertura assistencial em atenção primária e saúde mental, tendo como categorias de análise as regiões de saúde de cada estado, indicando as contradições da oferta, silenciamentos quanto à fragilidade da retaguarda assistencial e os riscos diante do cenário de mudança e desmonte das políticas empreendidos nos últimos cinco anos.

\section{Resultados e Análise}

\section{Regiões de Saúde do Ceará, Piauí e Rio Grande do Norte segundo condições socioeconômicas e de oferta de serviços de saúde}

O Ceará possui 184 municípios que estão organizados em 22 diferentes regiões de saúde e 4 grupos de regiões de saúde, segundo condições socioeconômicas e de saúde. Dentre as regiões, 19 (86\%) fazem parte do Grupo 1 de indicadores de saúde (baixo desenvolvimento socioeconômico e baixa oferta de serviços); 01 (Maracanaú, área metropolitana) faz parte do Grupo 2 (médio/alto desenvolvimento socioeconômico e baixa oferta de serviços); 01 (Juazeiro do Norte) faz parte do Grupo 3 (médio desenvolvimento socioeconômico e média/ alta oferta de serviços) e 01 (Fortaleza, capital) faz parte do Grupo 5 (alto desenvolvimento socioeconômico e alta oferta de serviços). Tem destaque o fato de que 166 (90,2\%) dos municípios cearenses estão em território com baixo desenvolvimento socioeconômico e baixa oferta de serviços (Grupo 1). A população desse grupo corresponde a mais da metade da população do estado $(58,3 \%)$, indi- 
cando o baixo desenvolvimento e as precárias condições socioeconômicas em que vive a maior parte dos cearenses.

O estado do Piauí, por sua vez, possui 224 municípios, que dividem-se em 11 regiões de saúde, das quais $10(90,9 \%)$ fazem parte do Grupo 1 de indicadores de saúde (baixo desenvolvimento socioeconômico e baixa oferta de serviços) e 1 (região Entre Rios) faz parte do Grupo 3 (médio desenvolvimento socioeconômico e média/alta oferta de serviços), onde situa-se a capital Teresina. Dessa forma, 193 (86,1\%) dos municípios piauienses estão em território com baixo desenvolvimento socioeconômico e baixa oferta de serviços, o que sinaliza um cenário de dificuldades e realidades com demandas complexas que atinge grande parte da população piauiense $(62,4 \%)$, ou seja, mais de 2 milhões de habitantes, de acordo com a estimativa do DAB em 2018.

Quanto ao Rio Grande do Norte, destaca-se que, dos 167 municípios do estado, 95,81\% são de pequeno porte (com menos de 50 mil habitantes), sendo que a maior deles tem baixo desenvolvimento socioeconômico e baixa oferta de serviços. Das 8 regiões de saúde, 6 pertencem ao Grupo 1 de indicadores de saúde (baixo desenvolvimento socioeconômico e baixa oferta de serviços), 1 pertence ao Grupo 3 (médio desenvolvimento socioeconômico e média/alta oferta de serviços) e 1 ao Grupo 5 (alto desenvolvimento socioeconômico e alta oferta de serviços). Destaca-se que $88,62 \%$ dos municípios, dos quais reúnem $47,74 \%$ da população do estado, estão localizados no grupo de menor desenvolvimento e oferta de serviços (Grupo 1), enquanto a única região de saúde classificada como de alto desenvolvimento, $7^{\mathrm{a}}$ região (Metropolitana), em que se localiza a capital, tem o equivalente a $2,99 \%$ dos municípios e $38,36 \%$ do contingente populacional.

\section{Panorama da APS e da RAPS do Ceará, Piauí e Rio Grande do Norte}

Quanto às equipes de APS no Ceará, destacase que estão majoritariamente distribuídas pelo interior do estado $(85,4 \%)$ e em municípios de pequeno porte $(46 \%)$. No Piauí, todos os municípios do estado contam com a presença de ACS e equipes de Saúde da Família, 96,7\% dos municípios contam com pelo menos uma equipe do NASF e, assim como no Ceará, as estratégias de atenção primária em saúde (ACS/ESF/NASF) estão concentradas majoritariamente no interior do estado. O Rio Grande do Norte segue a mes- ma tendência; dos 167 municípios, 160 (95,81\%) possuem pelo menos uma equipe de NASF e todos possuem agentes comunitários de saúde e estratégia de saúde da família cadastrados. Assim, é possível dizer, conforme os dados do DAB de 2018, que estas estratégias da APS alcançavam praticamente todos os municípios potiguares naquele ano.

Os estados do Ceará, Piauí e Rio Grande do Norte juntos somavam, em 2018, 28.060 Agentes Comunitários de Saúde (ACS), 5.001 equipes de Estratégia de Saúde da Família (ESF) e 742 equipes de Núcleo Ampliado de Saúde da Família (NASF). Em todas essas estratégias, o Ceará possui os maiores quantitativos de equipes e o Rio Grande do Norte, os menores. Entretanto, o número de equipes está associado às especificidades dos processos de expansão e regionalização da APS em cada estado, à extensão territorial e ao contingente populacional assistido. Por esse motivo, na análise da oferta de dispositivos da APS, torna-se importante comparar as porcentagens de habilitação das equipes em relação ao teto de possibilidades, segundo os parâmetros estabelecidos pelo Ministério da Saúde (MS). Para este estudo foram considerados os parâmetros estabelecidos pelo MS pela Portaria $\mathrm{n}^{\circ} 2.488$, de 21 de outubro de 2011, uma vez que se trata de tomar em análise a expansão e regionalização da cobertura num período que coincide com vigência desta normativa. Tomando por referência estes parâmetros e proporcionalidades, descrevemos os dados de cada estado na Tabela 1.

Ao observarmos os dados dos três estados na Tabela 1 quanto ao percentual de equipes credenciadas em relação ao teto possível, percebe-se que, embora com pequenas diferenças entre si, os percentuais de habilitação alcançam em torno de $60 \%$ ou mais do teto, o que deriva em boas coberturas da ESF. Quanto aos percentuais de cobertura das equipes nos três estados investigados, de acordo com os dados da Sala de Apoio à Gestão Estratégica do SUS, o CE (79,84\%), PI $(99,73 \%)$ e RN $(77,07 \%)$ estão acima da cobertura nacional (64,19\%), enquanto somente o RN encontra-se um pouco abaixo da cobertura da região Nordeste $(79,31 \%)$. Além disso, as diferenças do Piauí em comparação ao Ceará e ao Rio Grande do Norte se repetem quando observada na Tabela 1 a proporção de ACS credenciados em relação ao teto estabelecido pelo Ministério da Saúde: $89,3 \%$ no PI, $67,49 \%$ no CE e $66,39 \%$ no RN. Pode-se observar também que os percentuais de NASF cadastrados nos três estados, em relação ao máximo de habilitações possíveis para 
Tabela 1. Porcentagem de habilitações de APS por estado, capital/interior e grupos de regiões de saúde por condições socioeconômicas e de oferta de serviços em relação ao teto.

\begin{tabular}{crrrrrr}
\hline & No ACS & \% ACS & No ESF & \% ESF & No NASF & \% NASF \\
\hline RN & 5.767 & 66,39 & 1.064 & 61,29 & 182 & 63,86 \\
Grupo 1 & 3.743 & 90,30 & 696 & 84,16 & 150 & 71,09 \\
Grupo 3 & 900 & 75,00 & 142 & 58,68 & 18 & 56,25 \\
Grupo 5 & 1.118 & 33,54 & 226 & 33,88 & 14 & 33,33 \\
Capital & 2.194 & 23,79 & 104 & 23,69 & 3 & 15,00 \\
Interior & 6.492 & 80,79 & 960 & 74,02 & 179 & 67,55 \\
CE & 15.122 & 67,49 & 2.586 & 67,5 & 295 & 62,77 \\
Grupo 1 & 11.055 & 84,57 & 1855 & 70,94 & 224 & 68,09 \\
Grupo 2 & 784 & 58,77 & 165 & 61,57 & 19 & 65,52 \\
Grupo 3 & 849 & 80,63 & 144 & 68,25 & 20 & 68,97 \\
Grupo 5 & 2.434 & 35,04 & 422 & 30,38 & 32 & 38,55 \\
Capital & 2.202 & 33,75 & 365 & 27,97 & 26 & 35,62 \\
Interior & 12.920 & 81,35 & 2.221 & 69,89 & 269 & 67,76 \\
PI & 7.171 & 89,30 & 1.351 & 84,6 & 265 & 67,95 \\
Grupo 1 & 4.897 & 97,78 & 919 & 92,64 & 226 & 77,13 \\
Grupo 3 & 2.274 & 75,25 & 432 & 71,52 & 39 & 40,21 \\
Capital & 1.378 & 65,03 & 263 & 62,03 & 3 & 5,77 \\
Interior & 5.793 & 98,00 & 1.088 & 92,83 & 262 & 77,51 \\
\hline
\end{tabular}

Fonte: Departamento de Atenção Básica.

cada município/estado, se aproximam, embora o Piauí tenha leve vantagem.

A análise proporcional da quantidade de NASF em relação ao máximo que cada estado comporta difere das anteriores, uma vez que a possibilidade de habilitação destas equipes depende da quantidade de ESF que os municípios possuem. Nesta direção, destaca-se que, no seu conjunto, os municípios do RN concretizaram apenas $63,86 \%$ da sua capacidade de habilitação de NASF, considerando-se o número de ESF cadastradas e o número mínimo de equipes a serem vinculadas a cada modalidade de NASF, conforme parâmetros do Ministério da Saúde. Este dado sugere uma presença ainda insuficiente das equipes multiprofissionais na atenção primária do RN. A região que possui o melhor resultado neste aspecto é a $1^{\text {a }}$ região (São José de Mipibu) que possui $82,50 \%$ da sua capacidade de habilitação efetivada, totalizando 33 NASF habilitados; já a que possui o pior resultado é a $7^{\text {a }}$ região ou região Metropolitana (33,33\%), puxada pela baixa presença de NASF na capital.

Apesar de todos os municípios do Piauí possuírem NASF e da maior parte das regiões apresentarem altas coberturas de ESF, observa-se que conjuntamente os municípios desse estado só integralizaram $67,9 \%$ das equipes de NASF das quais poderia dispor de acordo com o número de equipes de ESF credenciadas. Nas regiões de baixo desenvolvimento socioeconômico e oferta de serviços (Grupo 1), a maior integralização encontrada foi de $89,5 \%$ na região Cocais e a menor, 62,5\%, em Tabuleiros do Alto Parnaíba. Mais uma vez a região Entre Rios, que conta com a capital do estado, é um destaque negativo na cobertura de serviços da APS e, também nesse parâmetro, possui a menor porcentagem de NASF em relação ao critério mínimo, 40\%. Nesse indicador, os números são de forma geral inferiores às estratégias analisadas anteriormente, o que pode indicar uma maior dificuldade de implantação do NASF nesses municípios em relação à ESF e à EACS.

No Ceará, 178 municípios contam com pelo menos um NASF, porém, em sua totalidade, os municípios consolidaram apenas $67,5 \%$ de sua capacidade de acordo com o número de equipes de ESF no estado. O único grupo de regiões que não contam com 100\% dos municípios assistidos por essa estratégia é o Grupo 1, onde 5 regiões não conseguiram implantar NASF em todos os municípios. Entre as regiões do Grupo 1, o maior número para esse indicador foi de $92,3 \%$ na região de Acaraú, e o menor encontrou-se em Cascavel (55\%). Nota-se que esses números são bastante heterogêneos neste grupo, onde grande parte das regiões registra números entre 60 e $70 \%$ e outra 
gira em torno dos $90 \%$. Os grupos de regiões 2 e 3 apresentam coberturas bastante semelhantes nesse quesito e aproximam-se do número registrado pelo estado, no entanto a região do Grupo 1 (Fortaleza) apresenta um resultado aquém das demais regiões. A $21^{\text {a }}$ região (Juazeiro do Norte) apresenta $65,5 \%$ de sua capacidade de habilitação e a 2a (Maracanaú), 69\%, enquanto a região que comporta a capital alcança apenas $38,5 \%$.

Posto isso, ao compararmos os dados dos três estados quanto à porcentagem de equipes credenciadas em relação ao teto possível, percebe-se que o Piauí é, proporcionalmente, o estado com maior cobertura $(86,54 \%)$ de ESF, seguido do Rio Grande do Norte $(61,29 \%)$ e do Ceará (57,68\%), que apesar de possuir mais equipes é o que possui menor cobertura. A disparidade do Piauí em comparação ao Ceará e ao Rio Grande do Norte se repete quando observada a proporção de ACS credenciados em relação ao teto estabelecido pelo MS: $89,3 \%$ contra $67,49 \%$ no CE e $66,39 \%$ no RN. Pode ser observado também, no que diz respeito à porcentagem de NASF cadastrados em relação ao máximo de habilitações possíveis para cada município/estado, que o $\mathrm{CE}$, RN e PI têm percentuais semelhantes, embora o Piauí siga possuindo o melhor número entre os três: Piauí com $67,94 \%$ da capacidade de habilitação de NASF concretizada, Rio Grande do Norte $63,86 \%$ e Ceará $62,76 \%$.

A análise da Tabela 1 permite observar também a discrepância quando se compara o interior e a capital do estado nos três estados participantes deste estudo, de modo que as capitais figuram com piores índices de cobertura de APS. Os dados disponibilizados pelo DAB revelam ainda que as regiões de saúde enquadradas no grupo 1, com baixo desenvolvimento socioeconômico e baixa oferta de serviços, apresentam melhores coberturas de APS do que as regiões caracterizadas por melhores condições socioeconômicas e de oferta de serviços, grupos 3 e 5 , em todos os estados, sendo que as regiões de Natal-RN e Fortaleza-CE, ambas do grupo 5, apresentam os piores resultados. Em todos os estados, as regiões com maior vulnerabilidade na cobertura assistencial pela Atenção Primária em Saúde foram aquelas onde se situam as capitais.

Os números dos três estados apontam para uma interiorização bem-sucedida das estratégias de Agentes Comunitários de Saúde e Saúde da Família, enquanto os Núcleos Ampliados de Saúde da Família avançaram menos em termos de cobertura. O NASF é a estratégia com menores índices de habilitação e, portanto, menores co- berturas, o que para a atenção em saúde mental podemos considerar como um vetor de vulnerabilização dos cuidados no território. Destaca-se, no entanto, a importância dos NASF nesses cenários, para apoiar e qualificar o trabalho das ESF e ACS quanto ao cuidado na lógica da atenção psicossocial, uma vez que "o apoio matricial pode ser uma ferramenta facilitadora da acessibilidade dos usuários de saúde mental aos serviços de saú$\mathrm{de}^{\prime 10}$ (p.2165).

Quanto à atenção psicossocial estratégica, com base nos dados de 2018, verifica-se no Gráfico 1 a presença de serviços por estado e tipo de CAPS.

Ao compararmos o total de CAPS dos três estados, verifica-se que o estado do Ceará é o que possui maior quantidade destes serviços, mais que o dobro da quantidade de CAPS que possuem os estados do RN e PI. Entretanto, assim como os dispositivos da APS, os CAPS são habilitados atendendo a critérios populacionais, de modo que tanto o porte populacional dos municípios quanto o número total de municípios de um estado precisam ser considerados na análise das diferenças interestaduais. Neste sentido, utilizamos o indicador CAPS/100.000 hab., criado pelo Ministério da Saúde, para acompanhar a expansão da atenção psicossocial estratégica no Brasil.

Para melhor compreender o processo de regionalização da atenção psicossocial especializada e sua relação com a promoção da equidade, a partir do acesso, destaca-se o número e a cobertura de CAPS, considerando o recorte de grupos de regiões de condições socioeconômicas e de oferta de serviços de saúde, bem como por capital e interior (Tabela 2).

Assim, na expansão da Atenção Psicossocial Especializada, observa-se que a modalidade de Centro de Atenção mais presente é a de CAPS I, havendo poucos CAPS infanto-juvenis, álcool e drogas e de atenção $24 \mathrm{~h}$ nos três estados. No geral, as coberturas são altas, em comparação às nacionais (1 CAPS por 100.000 hab.), e satisfatórias em relação às da região Nordeste $(1,33)$, variando entre 0,93 e 1,27 CAPS por 100.000 habitantes. $O$ Rio Grande do Norte, que possuía uma taxa de cobertura de 0,64 em 2008, alcançou em 0,92 em 2014 e 0,93 em 2018; o Ceará, que figurava com 0,79 CAPS por 100.000 hab. em 2008, obteve em 2014 a taxa de 1,10 e, em 2018, 1,13; o Piauí, por sua vez, obtinha em 2008 uma cobertura de 0,58 CAPS por 100.000 hab., em 2014, 1,13 e em 2018, 1,27. Estes dados demonstram uma aceleração da expansão da Atenção Psicossocial Especializada 


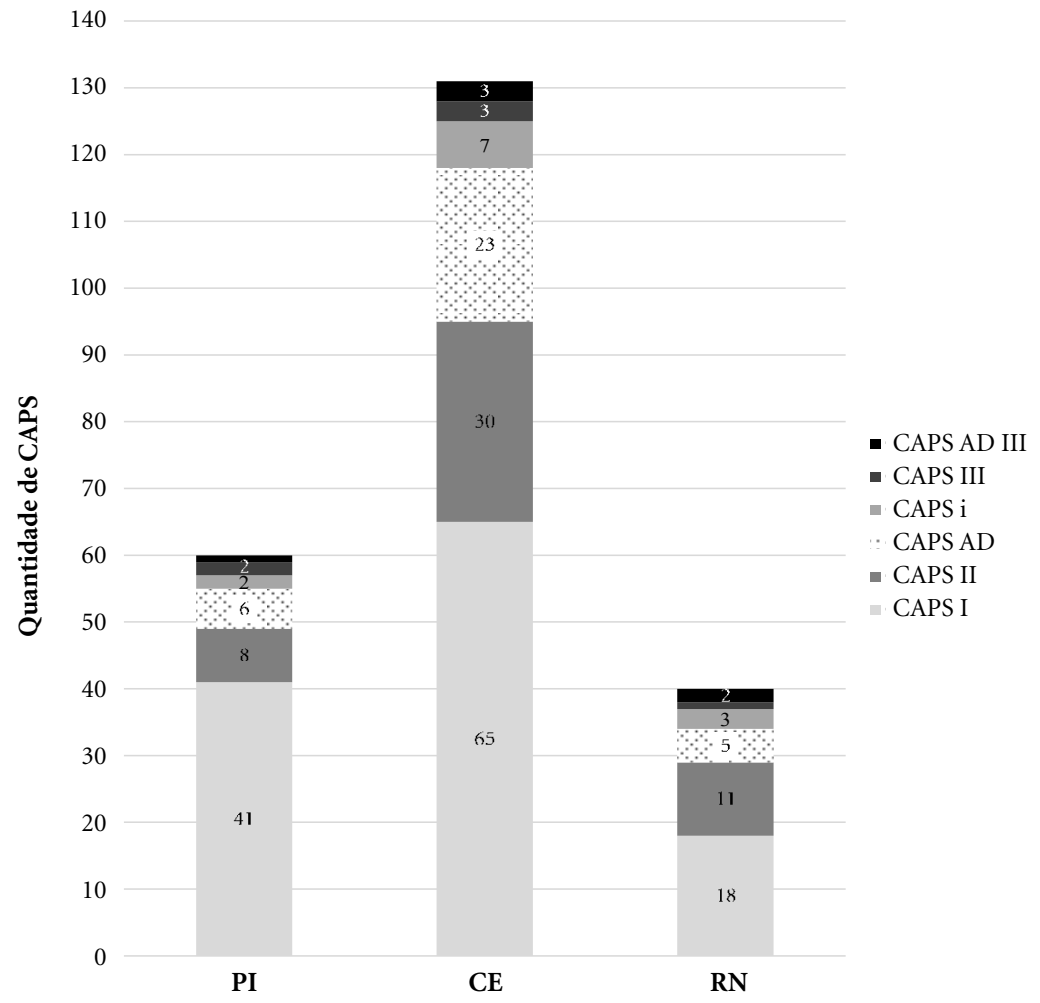

Gráfico 1. Quantitativo de CAPS por estado.

Fonte: Coordenação Nacional de Saúde Mental, Álcool e outras Drogas

nos três estados na última década, com uma leve desaceleração observada a partir de $2015^{11}$.

É possível observar também uma tendência à centralização dos serviços especializados de Atenção Psicossocial nas capitais e grandes municípios no interior dos estados, que parecem desempenhar o papel de referência para atenção psicossocial especializada em algumas regiões de saúde, embora as taxas de cobertura sigam baixas nestes locais, o que preocupa quanto à qualidade do cuidado ofertado, uma vez que parecem ser referência para grandes contingentes populacionais. Além disso, tendo em conta os limites das pactuações regionais entre municípios para organização das redes de atenção, marcadas por uma cultura de privilégio para negociação política em detrimento do planejamento ${ }^{12} \mathrm{e}$ as fragilidades de acesso colocadas pelas distâncias e dificuldades de transporte, não é possível garantir que esta cobertura derive em qualidade de acesso.

Verifica-se também que existem municípios sem CAPS cuja população supera o critério mí- nimo de implantação, enquanto outros possuem uma modalidade de CAPS habilitado embora sua população não seja suficiente para justificar sua implantação. Destas observações decorrem duas hipóteses sobre o modo como se deu a expansão da atenção psicossocial especializada no interior dos estados. A primeira é de que possa ter havido a habilitação de um mesmo CAPS de referência para um conjunto de municípios, atendendo o critério de população mínima, na lógica da criação de RAPS regionais; a segunda é de que o Ministério da Saúde possa ter flexibilizado os parâmetros populacionais de modo a viabilizar a interiorização da atenção psicossocial especializada. Para uma análise mais precisa acerca da abrangência populacional destes CAPS e das possibilidades de acesso ao cuidado, torna-se necessário verificar sua população de referência.

Observa-se ainda que, assim como acontece com os serviços de APS, os maiores vazios assistenciais quanto à cobertura de CAPS são encontrados nas regiões com melhores condições socio- 
Tabela 2. Quantitativo por tipo e cobertura de CAPS por estado, capital/interior e grupos de regiões de saúde por condições socioeconômicas e de oferta de serviços.

\begin{tabular}{crrrrrrr}
\hline & CAPSI & CAPSII & CAPSIII & CAPSAD & CAPSADIII & CAPSi & Cobertura \\
\hline RN & 18 & 11 & 1 & 5 & 2 & 3 & 0,93 \\
Grupo 1 & 13 & 4 & 1 & 1 & 1 & 0 & 0,87 \\
Grupo 3 & 4 & 2 & 0 & 1 & 1 & 1 & 1,51 \\
Grupo 5 & 1 & 5 & 0 & 3 & 0 & 2 & 0,78 \\
Capital & 0 & 2 & 0 & 2 & 0 & 1 & 0,56 \\
Interior & 18 & 9 & 1 & 3 & 2 & 2 & 1,05 \\
CE & 65 & 30 & 3 & 23 & 3 & 7 & 1,13 \\
Grupo 1 & 56 & 23 & 1 & 13 & 1 & 2 & 1,31 \\
Grupo 2 & 2 & 2 & 0 & 2 & 0 & 2 & 1,30 \\
Grupo 3 & 3 & 0 & 2 & 2 & 0 & 1 & 1,77 \\
Grupo 5 & 4 & 5 & 0 & 6 & 2 & 2 & 0,64 \\
Capital & 1 & 5 & 0 & 4 & 2 & 2 & 0,55 \\
Interior & 64 & 25 & 3 & 19 & 1 & 5 & 1,36 \\
PI & 41 & 8 & 1 & 6 & 2 & 2 & 1,27 \\
Grupo 1 & 32 & 5 & 0 & 5 & 2 & 1 & 1,49 \\
Grupo 3 & 9 & 3 & 1 & 1 & 0 & 1 & 0,91 \\
Capital & 0 & 3 & 1 & 1 & 0 & 1 & 0,76 \\
Interior & 41 & 5 & 0 & 5 & 2 & 1 & 1,46 \\
Nordeste & 996 & 111 & 13 & 66 & 18 & 33 & 1,33 \\
Brasil & 2256 & 393 & 59 & 254 & 57 & 155 & 1,00 \\
\hline
\end{tabular}

Fonte: Coordenação Nacional de Saúde Mental, Álcool e outras Drogas.

econômicas em todos os estados. Notadamente, as capitais apresentam os piores resultados neste indicador. Por outro lado, a baixa presença de CAPSad nos três estados contrasta com o alto número de Comunidades Terapêuticas, fazendo pensar sobre a relação entre a existência destas e a inibição da expansão da atenção psicossocial especializada em álcool e drogas. Os três estados possuem juntos 222 Comunidades Terapêuticas (CT) distribuídas, majoritariamente, em municípios do interior. Nos estados do Piauí e Ceará, as regiões de saúde que possuem o maior número de CT pertencem ao grupo de maior desenvolvimento e oferta de serviço nos estados, onde encontram-se as respectivas capitais. Já no Rio Grande do Norte, a região com maior concentração de CT está no grupo de menor desenvolvimento socioeconômico e não dispõe de nenhum CAPSad.

Quanto à atenção hospitalar, as taxas de cobertura dos leitos de saúde mental em hospital geral assemelham-se, sendo possível dizer que o número total de leitos dos três estados é bastante baixo: no RN, 1 leito de saúde mental para cada 319 mil habitantes; no CE, 1 leito para cada 358 mil habitantes; e, no PI, 1 para cada 323 mil habitantes. Portanto, é possível dizer que as inter- nações seguem ocorrendo majoritariamente em leitos de hospitais psiquiátricos.

No RN e PI, todos os leitos de saúde mental em hospitais gerais foram implantados nas capitais, já no CE, todos eles estão distribuídos em municípios do interior do estado em 4 diferentes regiões dos grupos 1 e 2. Embora todos os estados apresentem poucos leitos desse tipo, o Ceará obteve maior capilarização desse serviço no território do estado, enquanto no RN e PI observa-se uma centralização em apenas um município de referência.

Quanto aos leitos psiquiátricos, totalizam-se 1.079 leitos SUS nos três estados, observando-se um padrão de centralização nas regiões que comportam as capitais, onde se encontram as melhores condições socioeconômicas e maiores centros urbanos. O RN é, proporcionalmente, o estado com maior cobertura de leitos psiquiátricos, há uma proporção de cerca de 1 leito para cada 9 mil habitantes, enquanto CE e PI possuem, respectivamente, 1 para cada 16 mil e 1 para 20 mil.

Embora o modelo de atenção preconizado pela RAPS enfatize a desinstitucionalização, territorialização e integralidade do cuidado e das práticas em saúde mental, o grande contingente de leitos em hospitais psiquiátricos são flagran- 
tes da dificuldade de implementação desse novo modelo de atenção, em especial, no componente da atenção hospitalar. Tal cenário impõe a necessidade de ampliação da oferta de leitos de saúde mental em hospitais gerais, permitindo uma distribuição equitativa da atenção hospitalar ao longo das diversas regiões de saúde dos estados e, assim, uma maior capilaridade dos serviços hospitalares ofertados.

\section{Considerações sobre equidade e acesso aos cuidados em saúde mental em três estados nordestinos}

O cenário apresentado revela, no que se refere à equidade de cobertura e acessibilidade em saúde mental, que ainda precisamos avançar no país. Para Arakawa et al. ${ }^{13}$, a ampliação desses níveis depende de um necessário ajuste entre as necessidades da população e a oferta realizada. Nessa lógica, a equidade em saúde está ligada à qualidade dos serviços, isto é, sua eficácia e capacidade de resolver problemas ${ }^{14}$. Contudo, inúmeros obstáculos podem se colocar à ampliação e adequação da oferta, tais como barreiras estruturais, culturais e mesmo organizacionais ${ }^{15}$. No Brasil, como destacado anteriormente, estamos enfrentando um momento de grave fragilização institucional e crise política desde 2016, que vem reverberando no modo como o Ministério da Saúde tem operado. A população brasileira está enfrentando uma maior precariedade da rede de serviços e de pessoal no SUS, considerando o aprofundamento do desfinanciamento imposto pela política de austeridade fiscal e corte dos gastos públicos para o setor, nos últimos governos, especialmente em termos da atenção primária e psicossocial.

No entanto, pode-se observar que até dezembro de 2018, a política de regionalização da saúde, tomada como uma diretriz importante do Sistema Único de Saúde (SUS), produziu efeitos positivos na perspectiva de dar maior concretude ao princípio da equidade em saúde ao orientar a descentralização das ações e serviços, envolvendo processos de identificação das necessidades de saúde de cada território, por meio da análise da situação de saúde, planejamento e negociação e pactuação entre os gestores ${ }^{9}$.

As Regiões de Saúde foram estabelecidas como um recorte territorial ou espaço geográfico contínuo, constituído por agrupamentos de municípios limítrofes, com características culturais, econômicas e sociais semelhantes e redes de comunicação e infraestrutura de transportes compartilhados, com vistas a organização e qua- lificação do acesso e equidade das políticas públicas de saúde ${ }^{9}$. Assim cada região, por meio do trabalho das Comissões Intergestoras Regionais (CIR) tem a finalidade de integrar a organização, o planejamento e a execução de ações e serviços de saúde (atenção primária; urgência e emergência; atenção psicossocial; atenção ambulatorial especializada e hospitalar e vigilância em saúde), articulando-os pelas Redes de Atenção à Saúde: Rede Cegonha, Rede de Cuidado à Pessoa com Deficiência, Rede de Atenção Psicossocial, Rede de Urgência e Emergência e Rede de Atenção à Pessoas com Doenças Crônicas ${ }^{16}$.

No que se refere à saúde mental, detectouse nos três estados, que o processo de regionalização foi bem-sucedido, impactando em níveis satisfatórios de cobertura pelas equipes da atenção primária, à exceção do NASF, bem como de expansão dos dispositivos da RAPS, em especial, nas regiões com menor desenvolvimento socioeconômico e de oferta de serviços. Isso demonstra um importante processo de interiorização da atenção, pautado pelo princípio da equidade, na medida em que os vazios assistenciais vêm sendo progressivamente diminuídos nos territórios mais vulneráveis, possibilitando que um gradiente mais amplo de necessidades em saúde mental seja satisfeito. Ressalta-se, porém, que o componente da Atenção Hospitalar é o que mais deixa a desejar no que se refere ao processo de interiorização e expansão do acesso em hospitais gerais, desafio que se recrudesce nos cenários atuais de remanicomialização, ainda mais acompanhado com o desmonte das equipes de atenção primária, especialmente o NASF.

Por sua vez, é possível também identificar que as capitais e as áreas metropolitanas dos três estados, que possuem historicamente populações vulnerabilizadas em seus territórios periféricos, apresentam vazios assistenciais importantes, embora concentrem o maior número de serviços de APS/RAPS, conforme indicam seus índices de cobertura em comparação aos observados no interior. Entende-se que uma das razões para esta fragilidades nos padrões de equidade em saúde é a predominância, nessas áreas, do modelo de atenção focado na oferta de serviços especializados e, no que diz respeito à RAPS, o não fechamento dos hospitais e a grande oferta de leitos psiquiátricos. As capitais e grandes centros, de certo modo, segundo os dados de cobertura, parecem ter mais dificuldades para efetivar o cuidado territorial em saúde mental, perpetuando na oferta de serviços o modelo hospitalocêntrico e suas consequências cronificantes. Isso reverbera, 
consequentemente, na expansão do número de CT no interior dos estados e na dificuldade de atenção hospitalar nesses territórios.

Em se tratando de três estados situados na região nordeste do país, marcados historicamente pela precariedade das condições de vida e saúde da população, assim como por índices insatisfatórios de oferta de serviços, os dados apresentados demonstram que os investimentos feitos ao longo da última década nas políticas de atenção primária e de saúde mental perseguiram o princípio da equidade em saúde, produzindo efeitos em termos de ampliação do acesso pela população e de possibilidades do cuidado territorial, ao atingirem regiões anteriormente não contempladas em termos de atenção psicossocial.

Uma frente de avanço possível para o aprofundamento do princípio da equidade especialmente para qualificar a acessibilidade em saúde mental nos três estados, é o investimento nas equipes e gestores locais/regionais para elaborarem planos de ação que potencializem a atenção psicossocial no território de abrangência de cada região de saúde. Assim, é imprescindível investir no mapeamento e monitoramento dos casos de transtorno mental comum e do tipo grave e persistente, incluindo o comportamento suicida e de automutilação no território, especialmente aqueles que já são de conhecimento das equipes, além da população que faz uso de psicotrópico de forma continuada. O objetivo é elaborar de planos de cuidado, com foco no acompanhamento longitudinal e do trabalho em rede, observando a disponibilidade de oferta de atenção especializada com o acompanhamento do fluxo dos encaminhamentos do usuário na rede, além de avançar na formação de grupos de saúde mental e atividades comunitárias no próprio território ${ }^{17}$. Para isso, é urgente organizar as pactuações entre os diferentes pontos da Rede de Atenção à Saúde (RAS), a partir de sistemas de apoio técnico, logístico e de gestão, para garantir a integralidade no cuidado em saúde mental de forma regionalizada, diminuindo a demanda por internação em hospitais psiquiátricos, centralizados nas capitais, ou em comunidades terapêuticas, pulverizadas no interior. Mas não esqueçamos que esse cenário, duramente conquistado, está ameaçado pelos retrocessos políticos da conjuntura atual do Brasil, ampliando as desvantagens das populações de maior vulnerabilização social e aprofundando as iniquidades em saúde mental.

\section{Colaboradores}

M Dimenstein, ACR Simoni, JP Macedo e MTC Liberato participaram da concepção do tema do artigo, análise e interpretação dos dados, elaboração do texto e aprovação da versão final do escrito. N Nogueira, BCNS Barbosa, BIBM Silva, JB Amaral Filho, RCA Silva participaram da coleta e análise de dados e da redação do artigo. CLC Prado, MVAS Leão, BA Quinto e LF Soares contribuíram na coleta e interpretação dos dados. 


\section{Agradecimentos}

Ao Conselho Nacional de Desenvolvimento Científico e Tecnológico (CNPq), por meio das Bolsas de Produtividade em Pesquisa (PQ) e de Iniciação Científica (PIBIC).

\section{Referências}

1. Barros FPCD, Sousa MFD. Equidade: seus conceitos, significações e implicações para o SUS. Saude Soc 2016; 25(1):9-18

2. Paim JS. Sistema Único de Saúde (SUS) aos 30 anos. Cien Saude Colet 2018; 23(6):1723-1728.

3. Fournier J, Heale R, Rietze, LL. "I Can't Wait": Advanced Access decreases wait times in Primary Healthcare. Healthc Quarterly 2012; 15(1):64-68.

4. Cruz NFO, Gonçalves RW, Delgado PGG. Retrocesso da reforma psiquiátrica: o desmonte da política nacional de saúde mental brasileira de 2016 a 2019. Trab Educ Saude 2020; 18(3):e00285117.

5. Guimarães TA, Rosa LCDS. A remanicomialização do cuidado em saúde mental no Brasil no período de 2010-2019: análise de uma conjuntura antirreformista. O Social em Questão 2019; 22(44):111-138.

6. Ribeiro MKP, Gianini RJ, Goldbaum M, Cesar CLG. Equidade na cobertura dos gastos com saúde pelo Sistema Único de Saúde de pessoas com indicativos de transtornos mentais comuns no município de São Paulo. Rev Bras Epidemiol 2018; 21:e180011.

7. Viana ALÁ, Bousquat A, Melo GA, Negri Filho A, Medina MG. Regionalização e Redes de Saúde. Cien Saude Colet 2018; 23(6):1791-1798.

8. Duarte LS, Pessoto UC, Guimarães RB, Heimann LS, Carvalheiro JR, Cortizo CT, Ribeiro EAW. Regionalização da saúde no Brasil: uma perspectiva de análise. Saude Soc 2015; 24(2):472-485.

9. Viana ALD, Bousquat A, Pereira APCDM, Uchimura LYT, Albuquerque MVD, Mota PHDS, Demarzo MMP, Ferreira MP. Tipologia das regiões de saúde: condicionantes estruturais para a regionalização no Brasil. Saude Soc 2015; 24(2):413-422.

10. Quinderé PHD, Jorge MSB, Nogueira MSL, Costa LFA, Vasconcelos MGF. Acessibilidade e resolubilidade da assistência em saúde mental: a experiência do apoio matricial. Cien Saude Colet 2013; 18(7):21572166.

11. Brasil. Ministério da Saúde (MS). Saúde Mental em Dados - 12. Brasília: MS; 2015.

12. Mello GA, Pereira APCM, Uchimura LYT, Iozzi FL, Demarzo MMP, Viana, ALÁ. O processo de regionalização do SUS: revisão sistemática. Cien Saude Colet 2017; 22(4):1291-1310.

13. Arakawa T, Magnabosco GT, Brunello MEF, Andrade RLDP, Saita NM, Monroe AA, Villa TCS. Contextos locais e dos sistemas de saúde no controle da tuberculose, estado de São Paulo. REME 2020; 24:e-1296.

14. Campos GWS. Reflexões temáticas sobre equidade e saúde: o caso do SUS. Saude Soc 2006; 15(2):23-33.

15. Vieira-da-Silva LM, Esperidião MA, Viana SV, Alves VS, Lemos DVS, Caputo MC, Cardoso MO, Chaves SCL, Souza LEPF, Santana EM, Oliveira MCBA, Cunha ABO. Avaliação da implantação de programa voltado para melhoria da acessibilidade e humanização do acolhimento aos usuários na rede básica: Salvador, 2005-2008. Rev Bras Saúde Matern Infant 2010 10(Supl. 1):S131-S143. 
16. Brasil. Decreto no 7.508 , de 28 de junho de 2011 . Regulamenta a Lei no 8.080 , de 19 de setembro de 1990 , para dispor sobre a organização do Sistema Único de Saúde - SUS, o planejamento da saúde, a assistência à saúde e a articulação interfederativa, e dá outras providências à Rede de Atenção à Saúde. Diário Oficial da União 2011, 28 jun.

17. Rotoli A, Silva MRS, Santos AM, Oliveira AMN, Gomes GC. Saúde mental na Atenção Primária: desafios para a resolutividade das ações. Esc Anna Nery 2019; 23(2):e20180303.

Artigo apresentado em 07/08/2020

Aprovado em 01/03/2021

Versão final apresentada em 03/03/2021

Editores-chefes: Romeu Gomes, Antônio Augusto Moura da Silva 